Review

\title{
Superstructured poly(amidoamine) dendrimer-based nanoconstructs as platforms for cancer nanomedicine: A concise review
}

\author{
Cong Song ${ }^{\mathrm{a}}$, Mingwu Shen ${ }^{\mathrm{a}}$, João Rodrigues ${ }^{\mathrm{b}}$, Serge Mignani ${ }^{\mathrm{b}, \mathrm{c}}$, Jean-Pierre Majoral ${ }^{\mathrm{d}, \mathrm{e}}$, Xiangyang Shi ${ }^{\mathrm{a}, \mathrm{b}, *}$ \\ a State Key Laboratory for Modification of Chemical Fibers and Polymer Materials, College of Chemistry, Chemical Engineering and Biotechnology, Donghua University, \\ Shanghai 201620, People's Republic of China \\ ${ }^{\mathrm{b}}$ CQM-Centro de Química da Madeira, Universidade da Madeira, Campus da Penteada, 9000-390 Funchal, Portugal \\ ${ }^{\mathrm{C}}$ Université Paris Descartes, PRES Sorbonne Paris Cité, CNRS UMR 860, Laboratoire de Chimie et de Biochimie Pharmacologiques et Toxicologique, 45, rue des Saints Peres, 75006 \\ Paris, France \\ d Laboratoire de Chimie de Coordination du CNRS, 205 route de Narbonne, BP 44099, 31077 Toulouse Cedex 4, France

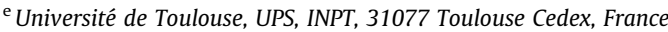

\section{A R T I C L E I N F O}

\section{Article history:}

Received 24 April 2020

Accepted 18 June 2020

Available online 6 July 2020

\section{Keywords:}

Dendrimers

Superstructures

Synthesis

Self-assembly

Cancer nanomedicine

\begin{abstract}
A B S T R A C T
Poly(amidoamine) (PAMAM) dendrimers, as a family of synthetic macromolecules with highly branched interiors, abundant surface functional groups, and well-controlled architecture, have received immense scientific and technological interests for a range of biomedical applications, in particular cancer nanomedicine. However, due to the drawbacks of single-generation dendrimers with a quite small size (e.g., generation 5 (G5) PAMAM dendrimer has a size of $5.4 \mathrm{~nm}$ ) such as limited drug loading capacity, restricted tumor passive targeting based on enhanced permeability and retention effect, and lack of versatility to render them with stimuli-responsiveness, superstructured dendrimeric nanoconstructs (SDNs) have been designed to break through these obstacles in their applications in cancer nanomedicine. Here, we review the recent advances related to the creation of SDNs such as dendrimer dumbbells, core-shell tecto dendrimers, dendrimer nanoclusters (NCs), dendrimer nanogels and dendrimer-templated hybrid NCs, and how these SDNs have been designed as nanoplatforms for different biomedical applications related to cancer nanomedicine including MR imaging, drug/gene delivery, combination therapy and theranostics. This review concisely describes the latest key developments in the field and also discusses the possible challenges and perspectives for translation applications.
\end{abstract}

\section{Contents}

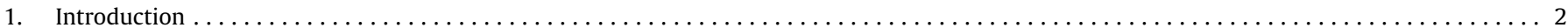

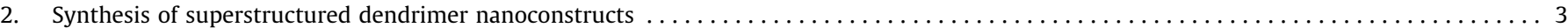

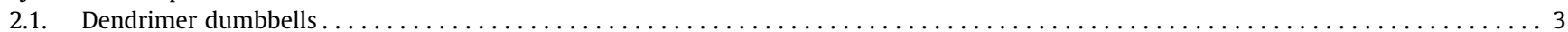

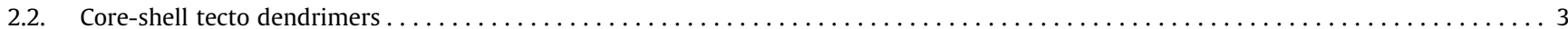

Abbreviations: Ad, adamantane; Ad-PEG, Ad-grafted polyethylene glycol; AFM, atomic force microscopy; AG, alginate; Au-DENs, dendrimer-entrapped gold nanoparticles: Au-DSNs, dendrimer-stabilized gold nanoparticles; Bcl-2, B-cell lymphoma 2; BM, benzimidazole; $\beta$-CD, $\beta$-cyclodextrin; CD-PEI, $\beta$-CD-grafted branched polyethylenimine; CSTDs, core-shell tecto dendrimer; DDs, dendrimer dumbbells; 3D MCTS, 3-dimensional multicellular tumor spheroids; 2D NOESY, two dimensional nuclear overhauser effect nuclear magnetic resonance spectroscopy; DNs, empty dendrimers; DOX, doxorubicin; DSP, 3,3'-dithiodipropionic acid-di(N-succinimidyl ester); DTPA, diethylenetriaminepentaacetic acid; ECH, epichlorohydrin; EDC, 1-ethyl-3-(3-dimethylaminopropyl) carbodiimide hydrochloride; EPI, epirubicin; EPR, enhanced permeability and retention; FA, folic acid; FITC, fluorescein isothiocyanate; Gd, gadolinium; ICG, indocyanine green; Luc, luciferase; MR, magnetic resonance; MTX, methotrexate; Mw, molecular weight; NCs, nanoclusters; NGs, nanogels; NHS, N-(e-maleimidocaproyloxy)-succinimide ester; NHS-PEG-NHS, polyethylene glycol-NHS ester; NPs, nanoparticles; PAMAM, poly(amidoamine); PCL, polycaprolactone; PCL-PAMAM/Pt, platinum prodrug conjugated PAMAM-graft-PCL; PEG-b-PAEMA, polyethylene glycol-b-poly(2-azepane ethyl methacrylate); PEI, polyethylenimine; Pt, platinum; PVDF, polyvinylidene fluoride; RAADyC, Ac-arg-ala-ala-asp-D-tyr-cys-NH ${ }_{2}$; $\mathrm{RGDC}$. Fmoc-arg-gly-asp-cys-SH; SAT $(\mathrm{PEG})_{4}$, N-succinimidyl (S)-acetyl(thiotetraethylene) glycol; SDNs, superstructured dendrimeric nanoconstructs; SDPs, supramolecular dendrimer particles; siRNA, small interfering RNA; SNPs, supramolecular nanoparticles; ZOL, zoledronic acid.

* Corresponding author at: State Key Laboratory for Modification of Chemical Fibers and Polymer Materials, College of Chemistry, Chemical Engineering and Biotechnology, Donghua University, Shanghai 201620, People's Republic of China.

E-mail address: xshi@dhu.edu.cn (X. Shi). 


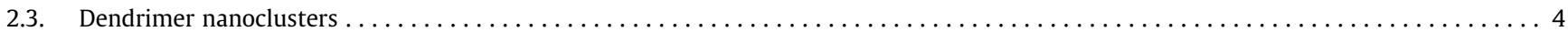

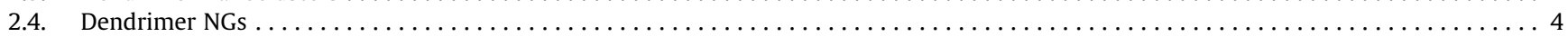

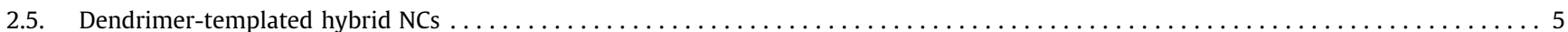

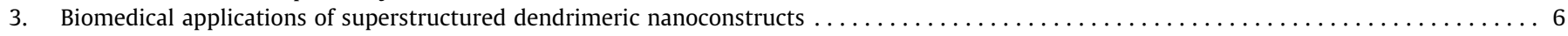

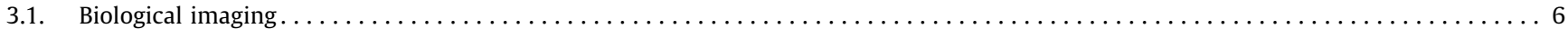

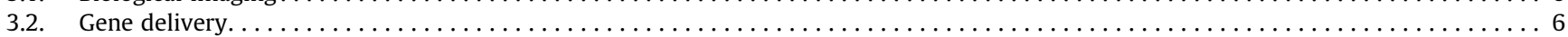

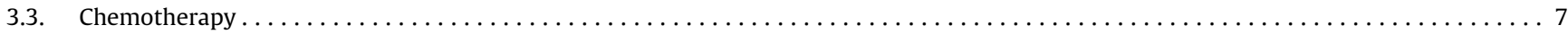

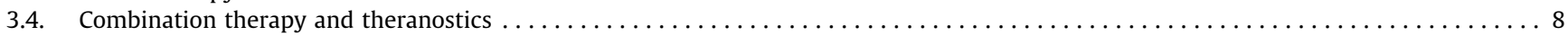

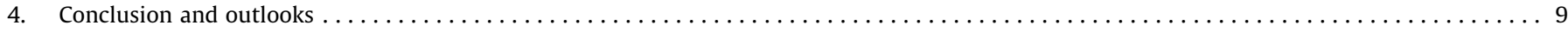

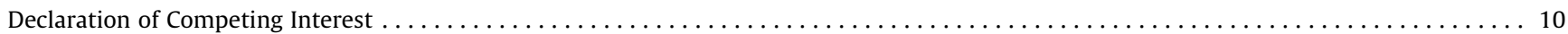

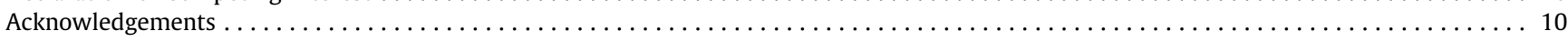

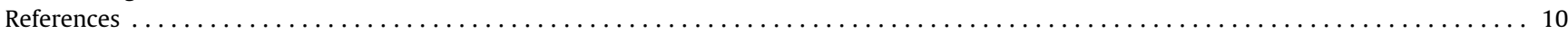

\section{Introduction}

Dendrimers, the term originated from the Greek word "dendrons", are recognized as a family of synthetic macromolecules with highly branched three-dimensional architectures and monodispersed characteristics [1-5]. Compared to conventional linear polymers, dendrimers, especially poly(amidoamine) (PAMAM) dendrimers, display some superiorities such as welldefined composition with good monodispersity close to proteins, abundant terminal functional groups, highly branched inner cavities, desired water-solubility, easiness of surface functionalization and non-immunogenicity [6-10]. Therefore, dendrimers have been extensively employed in the areas of biomedicine, in particular cancer nanomedicine due to their convincing assets. PAMAM dendrimers have been used as a platform to load different therapeutic drugs and/or diagnostic agents for drug/gene delivery [11-20], nuclear medicine [21-24], molecular imaging [25-34], and theranostics [35-38].

With the continuous and deep exploration of dendrimers for cancer nanomedicine applications, some limitations have been revealed. Generally speaking, high-generation dendrimers with a relatively large molecular dimension display a better gene transfection efficiency $[39,40]$, easier permeabilization of cell plasma membranes [41,42], and higher drug loading efficiency than lowgeneration counterparts, which nonetheless can be easily synthesized $[13,43]$. Moreover, gadolinium (Gd) chelate-modified dendrimers with a high molecular weight $(\mathrm{Mw})$ or large global dimension possess a higher $r_{1}$ relaxivity than those with a low Mw and small global dimension owing to the prolonged rotational correlation time of $\mathrm{Gd}(\mathrm{III})$, which is beneficial for improved magnetic resonance (MR) imaging sensitivity $[44,45]$. In most cases, high-generation dendrimers (G4 or above) are more suitable for practical biomedical applications than low-generation dendrimers, and the high-generation dendrimers, although commercially available, are expensive due to the tedious synthesis and purification steps and their inherent generational, skeletal, and substitutional diversities [5,46,47]. Furthermore, the size limit of singlegeneration dendrimers at a high generation (e.g., G8 PAMAM) is still smaller than $10 \mathrm{~nm}$, which cannot fully benefit from the enhanced permeability and retention (EPR)-based passive tumor targeting, thereby limiting their effective tumor therapy and imaging applications. Not to mention that, the drug loading capacity of single high-generation dendrimers still cannot be comparable to other formulations such as micelles, vesicles, liposomes, and nanogels (NGs) due to their small sizes and limited volumes of internal cavities. Furthermore, single-generation dendrimers except for the cystamine-cored PAMAM dendrimers with redox-responsiveness [48] hardly display stimuli-responsiveness, thereby limiting their desired biomedical applications, in particular in the circumstances related to tumor microenvironment-responsive delivery.
To overcome the limitation of the single-generation dendrimers, one of the feasible pathways is to use dendrimers as reactive modules or building blocks to construct structure-controllable nanoarchitectures of higher complexity and dimensions. In this review, we refer to these dendrimeric nanoarchitectures as superstructured dendrimeric nanoconstructs (SDNs). Based on the product structure or synthesis strategy, the SDNs are typically classified into five categories such as dendrimer dumbbells (DDs) [49,50], core-shell tecto dendrimers (CSTDs) [51-53], dendrimer nanoclusters (NCs) [54-56], dendrimer NGs [57,58], and dendrimer-templated hybrid NCs [59-61]. Importantly, when compared to the preparation of high-generation dendrimers, these SDNs with diverse frameworks can be easily prepared. For instance, dendrimers of two different generations with interactive terminal groups can be coupled or self-assembled to construct nanoscale CSTDs, showing similar structure to high-generation dendrimers [4,52]. Dendrimer-based micelles with multiple dendrimers contained in each micelle can be prepared via electrostatic interaction of cationic-neutral diblock copolymers and carboxylterminated PAMAM dendrimers to form supramolecular structures [62]. In addition, single dendrimers are also able to be crosslinked to prepare dendrimer NCs with defined size and dimension [54,56]. In brief, the SDNs are generally based on two or more dendrimers to form superstructured nanocontructs through different types of interactions or driving forces.

Besides the inherent larger size-associated advantages, the SDNs own the similar properties to single-generation dendrimers such as abundant terminal functional groups, hydrophobic interiors, and high water-solubility, hence arousing great interest for various applications in cancer nanomedicine, including bioimaging $[50,54]$, gene delivery $[63,64]$, chemotherapy $[65,66]$, combination therapy [67], and imaging-guided therapy [68]. For instance, amine-surfaced CSTDs formed through supramolecular assembly of G5 dendrimers as cores and G3 dendrimers as shells enabled much higher gene delivery efficiency than singe G5 or G3 dendrimers [63]. Dendrimer NCs formed via crosslinking of PAMAM dendrimers could be conjugated with $\mathrm{Gd}(\mathrm{III})$ chelates for enhanced MR imaging [25,55], and dendrimer NCs loaded with anticancer drugs could be utilized for accelerated drug penetration within tumors to achieve improved therapeutic efficacy [59,60]. Dendrimer NGs with excellent flexibility and versatility have been employed for tissue engineering, cancer imaging, and drug delivery $[58,65,69]$. Although various achievements have been made so far in the field, currently, there are no reviews specifically focused on the development of SDNs for cancer nanomedicine applications. Herein, we review the recent key achievements in the generation and biomedical applications of SDNs, with a focus on cancer nanomedicine. It should be noted that this is not an extensive review to cover all aspects of SDNs, but rather covers the recent key developments of SDNs for improved cancer nanomedicine applications. 
Therefore, aspects associated to dendron-related nanoarchitectures such as supramolecular hybrid dendrimers derived from low-generation peptide dendrons [70], binary dendrons [71], and amphiphilic dendron-assembled micelles [72-74], dendrimerstabilized [27,75-85], dendrimer-assembled [34,86-90] nanoparticles (NPs), or polydendrimers [91] not related to PAMAM dendrimers will not be covered here.

\section{Synthesis of superstructured dendrimer nanoconstructs}

The well-defined structure and controllable surface functionalities render dendrimers with promising reactive modules or building blocks to create versatile SDN-based platforms. Dendrimers have been used to construct supramolecular DDs, CSTDs, dendrimer NCs, dendrimer NGs or dendrimer-templated hybrid NCs. For the sake of clarity, Table 1 highlights various types of representative SDNs existing in the literature (Table 1).

\subsection{Dendrimer dumbbells}

Oligonucleotides have been deemed as outstanding tools for driving the self-assembly of dendrimers in precise structural arrangements due to the base specificity of the resulting duplex structure [49,92]. The oligonucleotide-mediated self-assembly of dendrimers to form DDs is quite simple and just requires only two conjugation steps $[50,93]$. In an earlier report, Baker and coworkers investigated DNA-directed synthesis of G7 and G5 PAMAM dendrimer DDs (G7-DNA-G5) [49]. Carbodiimide/imidazole chemistry was used to partially link complementary oligonucleotides (5'-phosphate-modified) to acetylated G7 and G5 PAMAM dendrimers, respectively. After that, equimolar amounts of the G5 and G7 conjugates were hybridized via complementary base pairing to afford the DNA-directed self-assembly of supramolecular DDs. Agarose gel electrophoresis was used to confirm the successful covalent linking of dendrimers with oligonucleotides, and atomic force microscopy (AFM) imaging was utilized to prove the formation of DDs.

Based on the easiness to generate oligonucleotide-conjugated dendrimer components, construction of combinatorial libraries of dendrimer-linked anticancer therapeutics, imaging, and targeting agents would be possible. In another noticeable study from the Baker group, DDs with functional molecules were built up [50]. G5 PAMAM dendrimers were respectively conjugated with fluorescein (FITC) and folic acid (FA), then linked together through hybridization of complementary DNA oligonucleotides to produce DDs of FITC-G5-DNA-G5-FA. The obtained FITC-G5-DNA-G5-FA DDs enabled targeted fluorescence imaging of cancer cells overexpressing the high-affinity FA receptor.

As mentioned, DNA-mediated DDs do have a number of advantages to be applied in the field of cancer nanomedicine. However, it is well advocated that the current method to synthesize DDs with multiple oligonucleotides on each dendrimer surface produces a statistical array of base-paired structures from which the desired product must be separated. Furthermore, a recent study showed that the same self-assembly process of DNA-mediated G3 DDs strongly depended on the charge of the dendrimer and length of the DNA used [94]. The DDs with non-protonated dendrimers could be easily prepared, and their inter-dendrimer distance strongly depends on the DNA length. In contrast, for complexes with protonated dendrimers, the inter dendrimer distance is independent of the DNA length, because the positively charged protonated dendrimers are combined with negatively charged DNA strands via electrostatic complexation. Hence, it is crucial to maintain the non-protonation of PAMAM dendrimers for the successful synthesis of DNA-dendrimer conjugates through a covalent manner for the subsequent generation of the DDs.

\subsection{Core-shell tecto dendrimers}

To avoid the tedious and time-consuming synthesis and purification of high-generation dendrimers, Tomalia and coworkers first initiated the synthesis of CSTDs [4,53]. In their work, amineterminated high-generation PAMAM dendrimers were used as cores (e.g., G5, G6, or G7) to react with low-generation carboxylterminated PAMAM dendrimers as shells (e.g., G1, G2, G3, or G4) via 1-ethyl-3-(3-dimethylaminopropyl) carbodiimide hydrochloride (EDC) coupling chemistry [52]. The formed individual CSTDs can be confirmed through AFM imaging to have increased dimension depending on the combination of different core/shell dendrimer components (i.e., G5/G3 < G6/G4 < G7/G5) [51]. For

Table 1

Summary of the formation of SDNs.

\begin{tabular}{|c|c|c|c|}
\hline Designations & Dendrimer-based monomer types & Interaction & Reference \\
\hline DDs & $\begin{array}{l}\text { G7 and G5 } \\
\text { FITC-G5 and G5-FA } \\
\text { G3 }\end{array}$ & $\begin{array}{l}\text { DNA-directed self-assembly via } \\
\text { complementary base }\end{array}$ & $\begin{array}{l}{[49]} \\
{[50,93]} \\
{[94]}\end{array}$ \\
\hline CSTDs & $\begin{array}{l}\text { Amine-terminated dendrimers (G5 to G7) and carboxyl- } \\
\text { terminated dendrimers ( } \mathrm{G} 1 \text { to } G 4 \text { ) } \\
\text { G5-CD and G3-Ad } \\
\text { G5.NHAc-CD and G3.NHAc-BM }\end{array}$ & $\begin{array}{l}\text { EDC coupling chemistry } \\
\text { Host-guest recognition of } \beta-C D \text { and } \mathrm{Ad} / \mathrm{BM}\end{array}$ & $\begin{array}{l}{[4,51-53,66,95]} \\
{[63,67]} \\
{[96]}\end{array}$ \\
\hline Dendrimer NCs & $\begin{array}{l}\text { G5 } \\
\text { G3 } \\
\text { G2 } \\
\text { G1 } \\
\text { Fluorodendrimers }\end{array}$ & $\begin{array}{l}\text { Crosslink reaction } \\
\text { Thiolation reaction } \\
\text { Crosslink reaction } \\
\text { Crosslink reactions in situ } \\
\text { Self-assembly }\end{array}$ & $\begin{array}{l}{[54]} \\
{[55]} \\
{[56,97]} \\
{[98]} \\
{[99]}\end{array}$ \\
\hline Dendrimer NGs & $\begin{array}{l}\text { G4-PEG/RGDC/RAADyC } \\
\text { Hydroxyl-terminated G5 } \\
\text { G3 or G5 } \\
\text { FITC-G5 } \\
\text { G3-Ad }\end{array}$ & $\begin{array}{l}\text { Oxidation reaction } \\
\text { Hydrophobic association } \\
\text { EDC chemistry } \\
\text { Emulsion method } \\
\text { Host-guest recognition of } \beta-C D \text { and Ad }\end{array}$ & $\begin{array}{l}{[57]} \\
{[58]} \\
{[65,69]} \\
{[68]} \\
{[100]}\end{array}$ \\
\hline Dendrimer-templated hybrid NCs & $\begin{array}{l}\text { G2 to G9 } \\
\text { Au-DENs, DNs or Au-DSNs } \\
\text { G6-P22 } \\
\text { PCL-PAMAM/Pt or PEG-b-PAEMA-PAMAM/Pt } \\
\text { G2-Ad }\end{array}$ & $\begin{array}{l}\text { Electrostatic interaction } \\
\text { Nanoprecipitation } \\
\text { Host-guest recognition of } \beta-C D \text { and Ad }\end{array}$ & $\begin{array}{l}{[62]} \\
{[101,102]} \\
{[103]} \\
{[59,60]} \\
{[104]}\end{array}$ \\
\hline
\end{tabular}


instance, G5/G3 CSTDs were found to have on the average $10 \mathrm{G} 3$ dendrimers surrounding each $\mathrm{G} 5$ dendrimer core, and the diameter of G5/G3 CSTDs was between those of G7 and G8 PAMAM dendrimers. Importantly, the G5/G3 CSTDs could be produced in only one step with a yield as high as $75 \%-83 \%$, whereas adding three generations to a G5 core by the divergent dendrimer synthesis method would involve six reaction steps [52]. Besides the formation of CSTDs using full-generation dendrimers, half-generation carboxylated PAMAM dendrimers can also be modified onto fullgeneration dendrimers through the same chemistry. For instance, G5/G2.5 CSTDs could be prepared using G5 PAMAM as core and G2.5 PAMAM as shell to have a hydrodynamic size close to that of G6.5 dendrimer [95]. In general, to ensure the successful generation of CSTDs, the core size of dendrimers must be larger than that of the shell dendrimer component to ensure the coverage of the core surface, and desired CSTDs can be quickly formed by selecting different dendrimer generations. Unfortunately, the generated CSTDs through EDC chemistry cannot be applied to synthesize CSTDs with amine surfaced shells by reacting carboxylated dendrimer core with the aminated dendrimer shell due to the technical problems related to the aggregation and precipitation.

To synthesize CSTDs with amine surface groups that can be better adapted for biomedical applications, our group has developed an original host-guest supramolecular assembly approach $[63,96]$. As an example (Fig. 1a), amine-terminated G5 and G3 PAMAM dendrimers were first modified with the host ( $\beta$ cyclodextrin, $\beta-C D$ ) and guest (adamantane, Ad) molecules, respectively. Through supramolecular recognition of $\beta-\mathrm{CD}$ and Ad moieties, G5-CD/Ad-G3 CSTDs were formed and confirmed by twodimensional nuclear overhauser effect nuclear magnetic resonance spectroscopy (2D NOESY) and AFM imaging to have a uniform morphology [63]. The facile non-covalent host-guest assembly can be extended to prepare pH-responsive CSTDs via host-guest recognition of $G 5-C D$ as a core and benzimidazole (BM)functionalized G3 dendrimers as shell components. Since the assembly between $\beta-\mathrm{CD}$ and $\mathrm{BM}$ is $\mathrm{pH}-$ responsive and can be dissociated under slightly acidic $\mathrm{pH}(\mathrm{pH}<6)$, the G5.NHAc-CD/BMG3.NHAc CSTDs could be adopted for $\mathrm{pH}$-responsive drug delivery applications [96].

\subsection{Dendrimer nanoclusters}

Unlike the preparation of DDs and CSTDs that have generally involved the use of dendrimers with two different generations, dendrimer NCs with a larger size can be prepared by crosslinking dendrimers of the same generation [54-56,98]. In an earlier report,
Tsourkas and coworkers created G5 dendrimer-based NCs (G5 NCs) by crosslinking amine-terminated G5 PAMAM dendrimers with bifunctional amine reactive polyethylene glycol (PEG)-NHS ester (NHS-PEG-NHS) [54]. The prepared G5 NCs had a good water solubility because of the hydrophilicity of the PEG spacers. In order to maintain an average hydrodynamic diameter of $150 \mathrm{~nm}$ with a relatively narrow size distribution, the optimal $\left[\mathrm{NH}_{2}\right] /[\mathrm{NHS}]$ molar ratio of 50: 1 was selected. In another work, the same group manufactured G3 NCs crosslinked through disulfide bonding [55]. As shown in Fig. 1b, the main steps were as follows: (1) G3 PAMAM dendrimers were reacted with N-succinimidyl (S)-acetyl (thiotetraethylene) glycol (SAT(PEG) 4 ) to synthesize thiolated dendrimers; (2) the thiolated dendrimers were suspended in an alkaline buffer for deprotection to form polydisulfide G3 NCs; and (3) after quenching of free thiols with maleimide, $\mathrm{Gd}(\mathrm{III})$ chelates were conjugated with the G3 NCs (Gd-G3 NCs) for further MR imaging applications. In this process, the size of G3 NCs increased with the extension of the reaction time. In order to obtain G3 NCs with various sizes, the unreacted thiols in an alkaline buffer could be quenched by adding excess maleimide at different reaction time points. The G3 NCs with average hydrodynamic diameters of 42 , 59, 91, 142 and $190 \mathrm{~nm}$ were prepared.

Besides the use of PEGylated crosslinkers, dendrimeric NCs have also been prepared using small molecular crosslinkers [56]. For instance, in a recent study by Cheng et al. [56], G2 NCs were synthesized by a disulfide-containing linker of 3,3'-dithiodipropionic acid-di(N-succinimidyl ester) (DSP). The produced G2 NCs had a relatively large hydrodynamic size around $40 \mathrm{~nm}$. In another report, Mamadou and co-workers synthesized low-generation PAMAM supramolecular dendrimer particles (SDPs) in situ in a catalytic polyvinylidene fluoride (PVDF) casting solution using amineterminated G1 PAMAM dendrimers $\left(\mathrm{G} 1 . \mathrm{NH}_{2}\right)$ crosslinked by epichlorohydrin (ECH) [98]. In brief, G1 and PVDF as homogeneous dispersion were mixed in triethyl phosphate at $80^{\circ} \mathrm{C}$, and crosslinked by $\mathrm{ECH}$. Through a phase inversion casting method, a flat SDP-containing sheet was formed with an average diameter of the PVDF-SDPs at $2.4 \mu \mathrm{m}$. The diameter of the PVDF-SDPs ranged from 0.7 to $3.3 \mu \mathrm{m}$, and it seemed that controlling the size of the dendrimer NCs was difficult via the developed method.

\subsection{Dendrimer NGs}

Unlike the difficulty to control the size of dendrimer NCs, dendrimer NGs, a type of nanoscale-sized hydrogel particles can have a better control of their sizes due to the formulation with the assistance of crosslinking agents, hydrophilic polymers or amphiphilic (a)

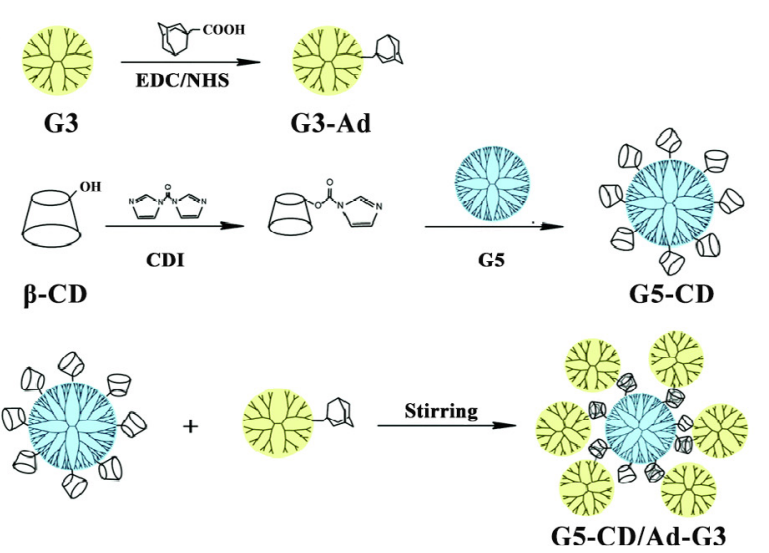

(b)
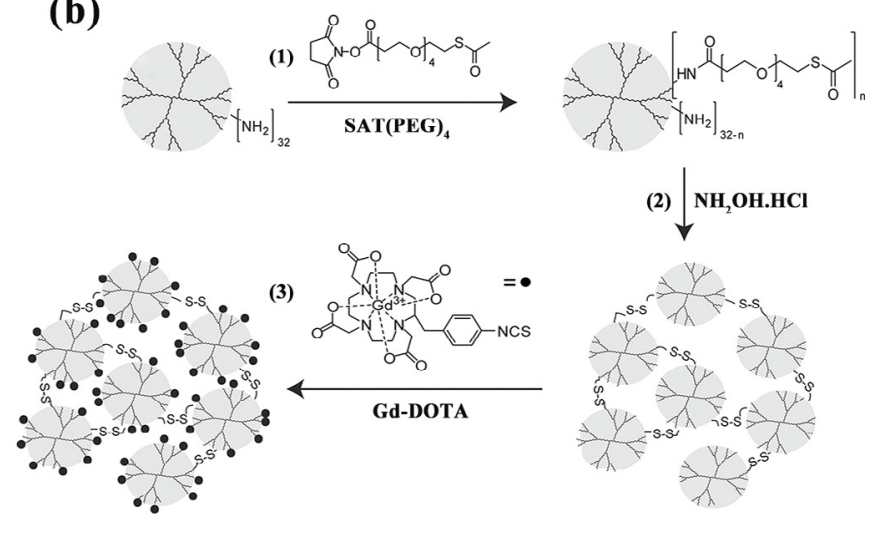

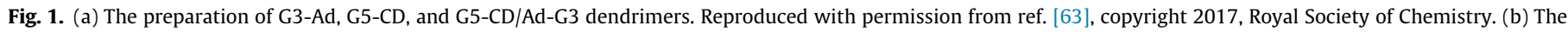
preparation of Gd-G3 NCs. Reproduced with permission from ref. [55], copyright 2012, American Chemical Society. 
surfactants to retain a significant amount of water. These dendrimer NGs possess several advantages, including good biocompatibility, ease of synthesis, and high drug loading capacity [57,58]. For instance, enzyme-sensitive dendrimer NGs loaded with drugs have been prepared through a chemical crosslinking method [57]. In this study, G4 PAMAM dendrimers modified with PEG2000-NHS were covalently conjugated with the bioadhesive components (Fmoc-arg-gly-asp-cys-SH, RGDC) and enzymeresponsive components (Ac-arg-ala-ala-asp-D-tyr-cys- $\mathrm{NH}_{2}$, RAADyC). Meanwhile, anticancer drug doxorubicin (DOX) was loaded within the dendrimer-based macromonomers. Then, the DOXloaded dendrimer NGs were constructed using sodium periodate to initiate the chemical crosslinking of the functional groups on the periphery of dendrimers. The resultant NGs were able to be decomposed in the presence of elastase to lead to the rapid release of drugs.

Different from Wang's work, Matai et al. [69] designed drugloaded alginate (AG)-G5 hybrid NGs through EDC coupling chemistry (Fig. 2). Three steps were developed to create the AG-G5 hybrid NGs: 1) AG carboxyl groups were first activated by EDC; 2) amine-terminated G5 dendrimers were then added dropwise into the AG solution to have increased viscosity, indicating the successful coupling between the dendrimer amine groups and the AG carboxyl groups; and 3) the mixture was then added with an anticancer drug epirubicin (EPI) to be encapsulated within the AG-G5 framework, followed by addition of divalent $\mathrm{Ca}^{2+}$ ions to crosslink the remaining AG carboxyl groups to form the EPI $\in$ AG-G5 NGs. The average hydrodynamic diameter of EPI $\in$ AG-G5 hybrid NGs $(360.7 \mathrm{~nm})$ was smaller than those of the single AG NGs (918.3 $\mathrm{nm}$ ) and drug-free AG-G5 NGs (417.6 nm), respectively, likely due to the increased crosslink densities of the starting materials. Alternatively, AG-G5 hybrid NGs could also be synthesized using an emulsion method to afford drug loading and imaging capability [68]. In this case, FITC-modified G5 PAMAM dendrimers were mixed with DOX and AG, double emulsified to form G5containing $\mathrm{AG} \mathrm{NGs}$, and added with $\mathrm{Ca}^{2+}$ ions to promote the crosslinking reaction, where both $\mathrm{CaCl}_{2}$ and $\mathrm{G} 5$ dendrimers acted as crosslinkers. It appears that $\mathrm{G} 5$ dendrimers could afford the
NGs with a compact morphology with a smaller size $(433 \pm 17 \mathrm{~nm})$ than those formed solely using the $\mathrm{Ca}^{2+}$ as a crosslinker $(873 \pm 116 \mathrm{~nm})$.

\subsection{Dendrimer-templated hybrid NCS}

Hierarchical control of molecules, macromolecules, and NPs is the central core of supramolecular and nanoscale chemistry [105-107]. Dendrimers or dendrimer-based NPs have been demonstrated to be excellent building blocks to construct hybrid SDNs. For example, a kind of particle-in-a-box-in-a-box system based on dendrimer-templated hybrid NCs has been constructed by Hove and co-workers [101]. As shown in Fig. 3a, G7 PAMAM dendrimer-entrapped gold nanoparticles (Au-DENs) were first formed by in situ reduction of Au salt precomplexed with dendrimers. The dendrimer-templated hybrid micelles (named dendrimicelles) were then assembled via mixing the Au-DENs at $\mathrm{pH}=7$ with an anionic-neutral block copolymer having a 64subunit polymethacrylic acid block and 885-subunit polyethylene oxide block. Simultaneously, other dendrimicelles with empty dendrimers (DNs) or mixed DNs and Au-DENs were also synthesized via the same process. TEM imaging revealed that each $\mathrm{Au}-$ DEN had an Au core size of $1.7 \mathrm{~nm}$ and each dendrimicelle had $23 \pm 7$ Au-DENs. In addition, as shown in Fig. 3b, the cryoTEM micrographs suggested that mixing empty and filled micelles did not seem to result in half-filled micelles, and the dendrimicelles still displayed the high stability even though they were left for 30 days at room temperature. Similarly, in another study of hove et al. [102], two kinds of dendrimicelles with supramolecular structure were manufactured using a congeneric mode through assembling G5 Au-DENs or G5 dendrimer-stabilized gold NPs (Au-DSNs). Among them, Au-DSNs refer to one Au NP surrounded by two or three dendrimers. In this case, the authors discovered that the formation of dendrimicelles with DENs or DSNs were dependent on the charge fraction (f), where $\mathrm{f}=$ (negative charge from the block copolymers)/(positive charge from the dendrimers). The dendrimicelles with explicitly defined nanostructures were able to be formed in the case of excess block copolymer to dendrimer

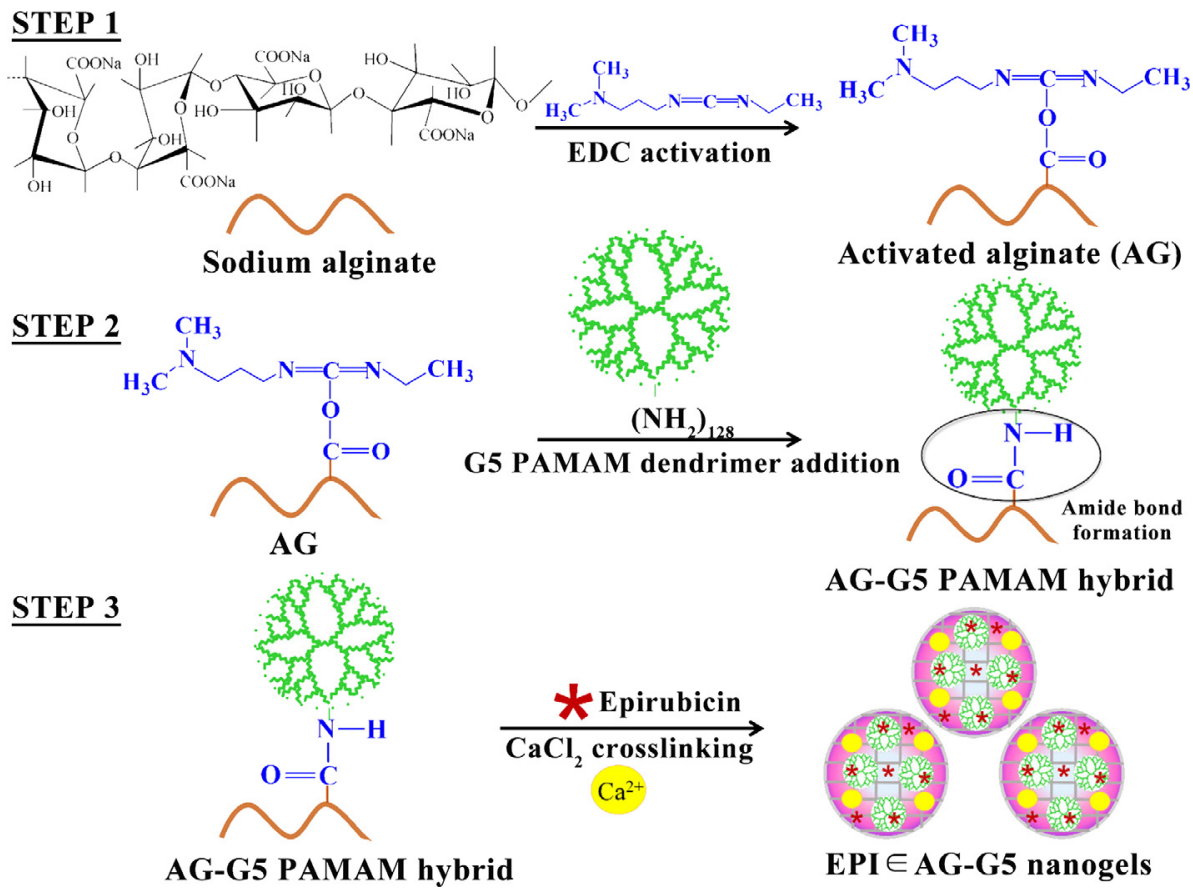

Fig. 2. Stepwise synthesis of EPI $\in$ AG-G5 NGs. Reproduced with permission from ref. [69], copyright 2016, American Chemical Society. 
(a)

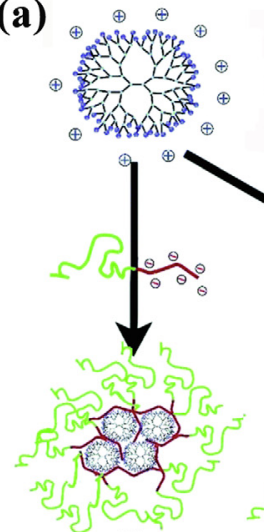

(1)
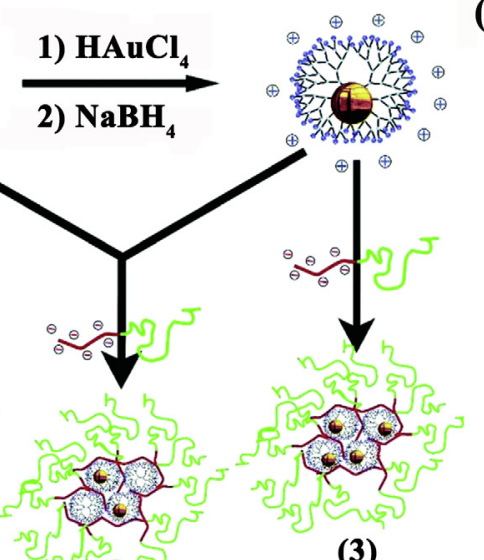

(2)

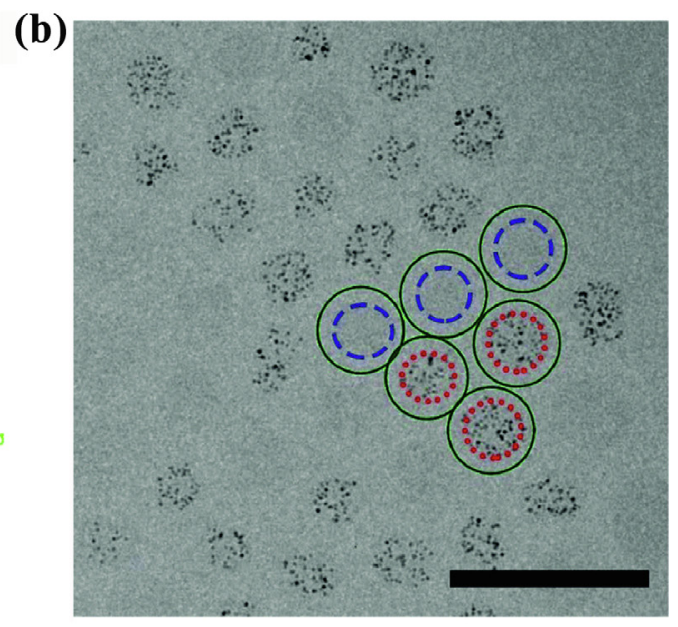

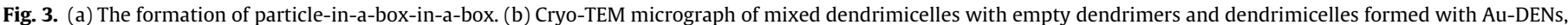

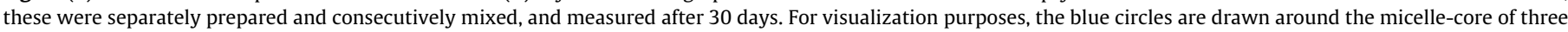

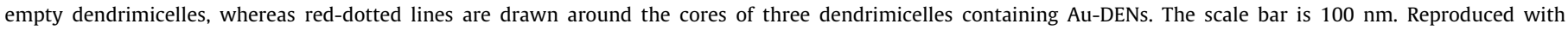
permission from ref. [101], copyright 2017, Royal Society of Chemistry.

( $\mathrm{f} \geq 1$ ), whereas excess dendrimer to block copolymer $(\mathrm{f}<1$ ) provided ill-defined nanostructures.

Different from those hybrid SDNs described above, Li et al. [59] established a smart polymeric clustered NP (iCluster) through coassembling polymer components including polycaprolactone (PCL) homopolymer, PEG-b-PCL copolymer and platinum (Pt) prodrug conjugated PAMAM-graft-PCL (PCL-PAMAM/Pt) via a nanoprecipitation method. In this process, PCL was chosen to control the size and stability of the iCluster, whereas PAMAM dendrimers were treated as a template to link these components through the reaction of the amine groups and the anhydride residue. In another work, Douglas et al. [103] employed G6 PAMAM dendrimers as templates to make bacteriophage P22 virus-like particles into an ordered array via electrostatic interaction. The ditopic capsid decoration protein acted as an auxiliary to cement P22 into place synchronously. It appeared that interparticle assembly occurred upon mixing P22 and G6 dendrimer at a molar ratio of 1:1000. Furthermore, the G6 dendrimers could be finally removed under a high ionic strength from the protein clusters.

\section{Biomedical applications of superstructured dendrimeric nanoconstructs}

Various SDNs have been synthesized for different biomedical applications in the field of cancer nanomedicine including biological imaging [50,55], gene delivery [56,63], chemotherapy [65,69], combination therapy [64], and theranostics [68]. Herein, we reviewed several key examples of the developed SDNs as nanoplatforms for cancer nanomedicine applications.

\subsection{Biological imaging}

Biological imaging is critical to characterize and measure the biological processes at the molecular or cellular level $[108,109]$. Nanoplatform-enabled bioimaging technology (e.g., fluorescence imaging and MR imaging) has become indispensable for tumor diagnosis. SDNs, as a unique nanoplatform, have been linked with fluorescent dyes or Gd(III) chelates for fluorescence or MR imaging of cancer cells or tumor tissues. As reported earlier by Baker and coworkers [50], DDs were designed with one dendrimer attached with targeting ligand FA and the other one linked with FITC for effective targeted imaging of FA receptor-expressing cancer cells in vitro. The DDs could be internalized across the cell membrane into the cancer cells by detecting and observing the FITC green fluorescence by confocal microscopy. Unfortunately, due to the limited penetration depth, the clusters have not been used for fluorescence imaging of a tumor model in vivo.

For MR imaging of tumors, dendrimer NCs formed through crosslinking of G5. $\mathrm{NH}_{2}$ dendrimers with NHS-PEG-NHS were used as a platform to sequentially link diethylenetriaminepentaacetic acid (DTPA) dianhydride for Gd(III) chelation, FA, and FITC (Fig. 4a) [54]. The multifunctional G5 NCs were applied for targeted fluorescence imaging of FA receptor-overexpressing cancer cells in vitro as confirmed by both flow cytometry and confocal microscopy. Moreover, due to enlarged molecular dimension of $\mathrm{Gd}(\mathrm{III})$ within the NCs to have prolonged rotational correlation time, the NCs displayed an $r_{1}$ relaxivity $\left(12.3 \mathrm{mM}^{-1} \mathrm{~s}^{-1}\right)$, much higher than Gd(III)-labeled individual G5 dendrimers $\left(10.1 \mathrm{mM}^{-1} \mathrm{~s}^{-1}\right)$ and the small molecular Gd-DTPA complex $\left(3.9 \mathrm{mM}^{-1} \mathrm{~s}^{-1}\right)$. The multifunctional NCs enabled enhanced targeted $\mathrm{T}_{1}$-weighted MR imaging of a xenografted tumor model, much better than the Gd(III)-labeled individual G5 dendrimers (Fig. 4b) presumably due to the larger size-induced improved EPR effect and $r_{1}$ relaxivity.

In another work, the same group prepared G3 NCs crosslinked through disulfide bonding and modified the G3 NCs with DOTA for $\mathrm{Gd}(\mathrm{III})$ chelation for $\mathrm{T}_{1}$-weighted $\mathrm{MR}$ imaging in vivo [55]. The authors showed that a significant contrast enhancement was seen in the kidneys and abdominal aorta at $15 \mathrm{~min}$ and $1 \mathrm{~h}$ postinjection, respectively. Quantitative analysis revealed a statistically significant MR enhancement in the kidneys for as long as $4 \mathrm{~h}$ when compared to the control group ( $\mathrm{p}<0.5$ ). In sharp contrast, Gd (III)-chelated individual G3 dendrimers did not reveal any enhancement in the kidneys at any of the time points. This illustrates the great advantage to use the NCs for better bioimaging applications than single dendrimers.

\subsection{Gene delivery}

Gene therapy based on transfection of DNA or RNA into targeted cells, has been extensively explored for cancer therapy [110]. In order to allow genes to quickly enter cells and function without being degraded, positively charged NPs are usually used as vectors to compact negatively charged genes for gene delivery to cells [111]. In principle, the transfection efficacy of high-generation PAMAM dendrimers is greater than the low-generation counter- 
(a)
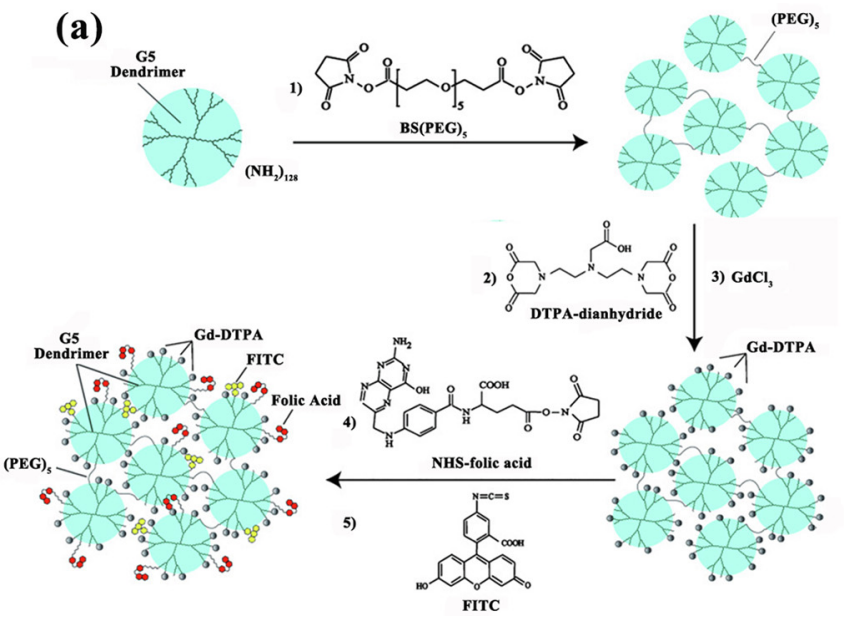

(b)

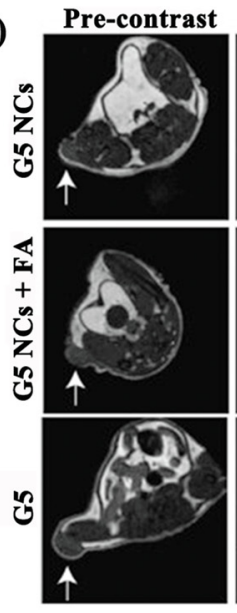

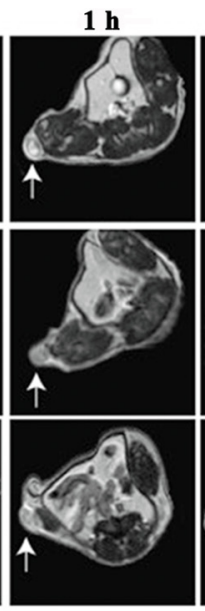

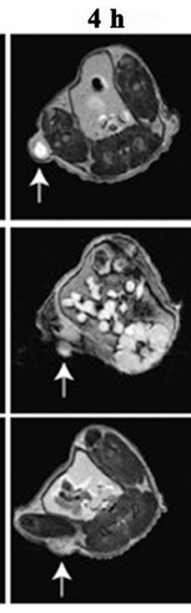

$24 \mathrm{~h}$

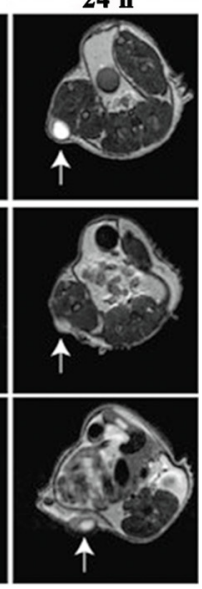

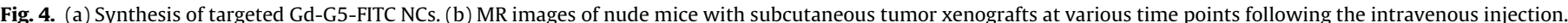

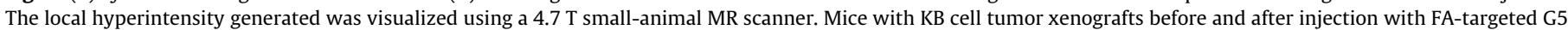

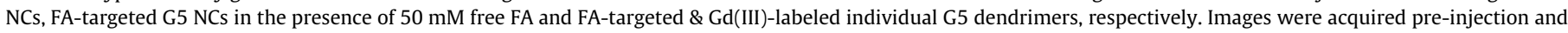

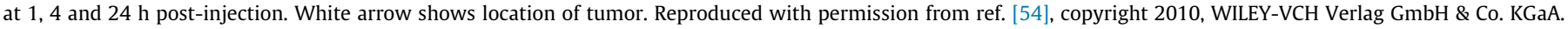

parts, while their cytotoxicity is higher [112,113]. Breaking up the correlation between transfection efficacy and cytotoxicity for dendrimer-based gene delivery system is urgent. In addition to the functional modification of dendrimers $[18,114,115]$, SDNs has been considered as one of the alternative solutions for highly efficient gene transfection with low toxicity. As demonstrated by Liu et al. [56], the G2 PAMAM dendrimers were utilized to obtain G2 NCs crosslinked with disulfide bond. Owing to the disulfide bond break in the endocytic pathway, the G2 NCs could degrade to form individual G2 dendrimers within cells, thus minimizing the damage of cationic single $\mathrm{G} 2$ dendrimers as a gene transfection vector to cells. Under an appropriate $\mathrm{N} / \mathrm{P}$ ratio (the molar ratio of primary amines of the dendrimers to phosphates in the pDNA backbone), the G2 NCs displayed comparable luciferase (Luc) gene expression efficacy to the gold standard of branched polyethylenimine (PEI) $(\mathrm{Mw}=25 \mathrm{kD})$, and superior efficacy than naked DNA, and single G2 or G5 dendrimers. In another study, Wang et al. [99] prepared dendrimer-based NCs via a self-assembly method. G1 and G2 dendrimers were first reacted with heptafluorobutyric anhydride to generate fluorodendrimers that were then self-assembled to form low-molecular-weight fluorodendrimer NCs with a spherical shape. The spherical NCs were used as vectors for gene transfection in the end. The results showed that the uniform NCs displayed a high transfection efficacy in vitro and in vivo, high permeability in 3-dimensional multicellular tumor spheroids (3D MCTS), minimal toxicity, and high serum stability, which presents a promising potential for clinical gene therapy applications.

Besides the dendrimer NCs, CSTDs with amine terminal groups synthesized in our previous work through supramolecular assembly were also used as a vector for gene delivery applications [63]. Our studies showed that the CSTDs with abundant surface amines on the surface were able to transfect the Luc gene with an efficiency 20 times and 170 times higher than single G5-CD and G3Ad dendrimers, respectively.

To render the cellular gene delivery with targeting specificity, SDNs have been modified with targeting ligands for enhanced and specific gene delivery. As reported by Wang et al. [104], the size-controllable RGD-targeted supramolecular nanoparticles (SNPs) were designed for gene delivery through four-step synthesis: (1) Ad-grafted G2 PAMAM (Ad-PAMAM), $\beta$-CD-grafted branched PEI (CD-PEI), and Ad-grafted PEG (Ad-PEG) were formed, respectively; (2) Ad-PEG was modified by RGD peptide; (3) the SNPs were synthesized through host-guest recognition based on
$\beta$-CD and Ad motifs; and (4) the anionic pDNA were compressed by cationic SNPs via electrostatic interactions. The results revealed that the SNP/DNA polyplexes had a satisfactory gene transfection efficiency in different cell lines such as U87, MCF-7 and 3 T3 cells. Furthermore, the SNP/DNA polyplexes enabled RGD-targeted specific gene delivery to cancer cells expressing integrin $\alpha_{v} \beta_{3}$.

\subsection{Chemotherapy}

NP-based drug delivery systems enable improved cancer chemotherapy by improving the pharmacokinetics and biodistribution of the anticancer drug and by controlling the release kinetics of the drugs to the target site [13]. As an ideal drug carrier, the SDNs with unique architectures have been employed as a carrier system for anticancer drug delivery applications [60,65,96]. Taking CSTDs as an example, G5/G2.5 CSTDs were used to load anticancer drug methotrexate (MTX) and zoledronic acid (ZOL) to treat melanoma cells in vitro [66]. In this study, 6 MTX and 31 ZOL molecules were loaded within the hydrophobic interior of the CSTDs (G5 cores), respectively, and the G2.5 shells were covered on the surface to improve the stability of the formulation. The obtained drug sandwiches showed a higher cytotoxicity to melanoma (Sk-Mel28) cells than both free drugs and G5/G2.5 CSTDs alone. At a certain MTX concentration (e.g., $50 \mu \mathrm{M}$ ), the MTX-sandwich could significantly inhibit the growth of Sk-Mel-28 cells without harming the keratinocytes.

The microenvironment of tumors can be considered as a battleground of a mixture of different molecules including lymphokines, cytokines, matrix metalloproteinase, collagen fibers, proteoglycans, and glycosaminoglycans, etc. To render the anticancer drug with tumor microenvironment-responsive delivery property, we developed original pH-sensitive supramolecular G5.NHAc-CD/ BM-G3.NHAc CSTDs for anticancer drug DOX delivery [96]. The hydrophobic interior of the CSTDs rendered them with a drug loading percentage of $5.16 \%$. By virtue of the $\mathrm{pH}$-sensitive assembly of $\mathrm{BM}$ and $\beta-\mathrm{CD}$ pair, the formed CSTDs/DOX complexes could be swallowed by cancer cells and dissociated to form singlegeneration dendrimers, enabling acidic $\mathrm{pH}$-triggered fast release of DOX. As shown in Fig. 5, the drug penetration depth and therapeutic efficacy of the CSTDs/DOX complexes were assessed in vitro via 3D MCTS, and the pH-insensitive CSTDs (G5.NHAc-CD/Ad-G3. NHAc)/DOX complexes were also compared. On one hand, the pH-sensitive CSTDs/DOX complexes displayed the strongest pene- 

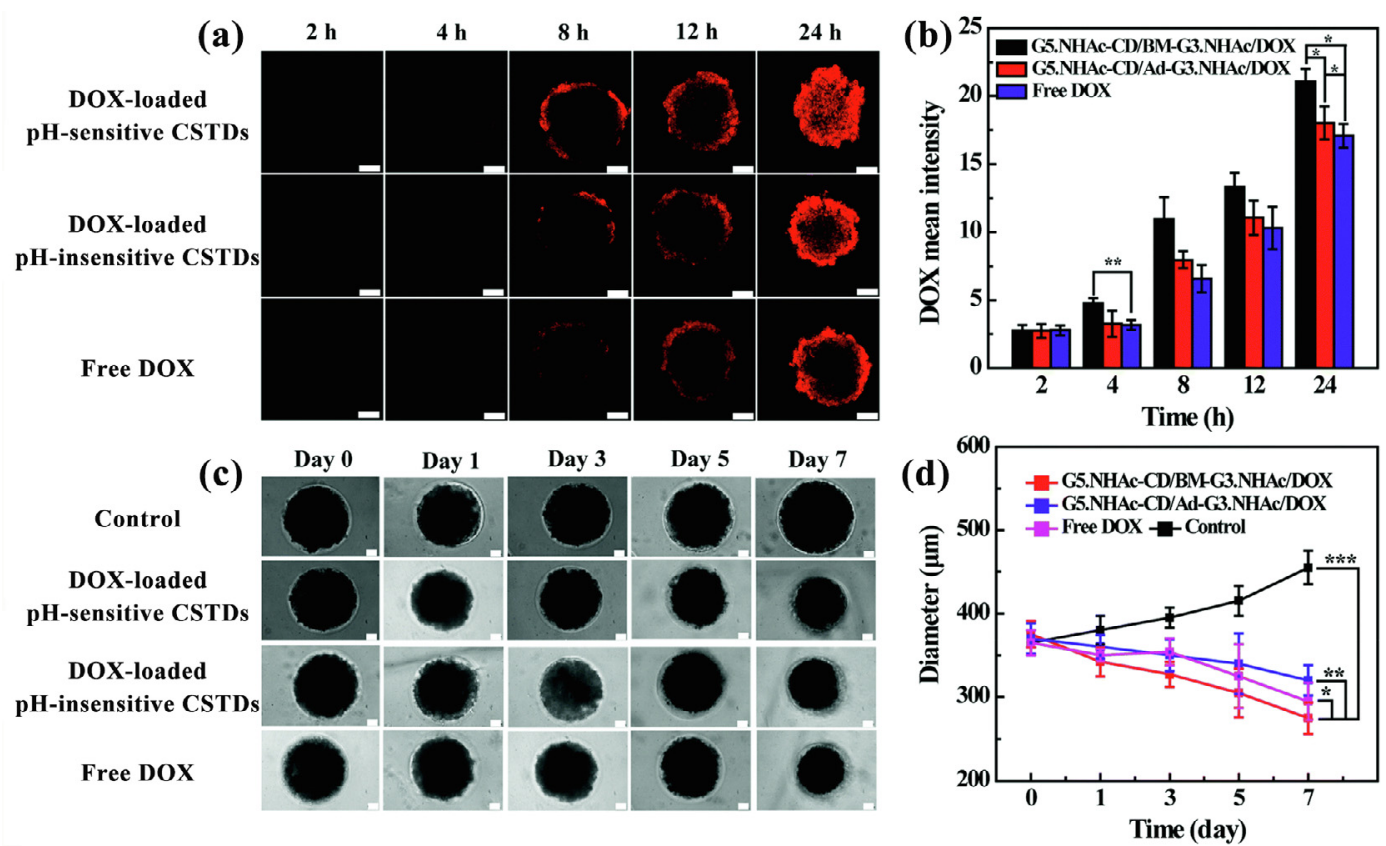

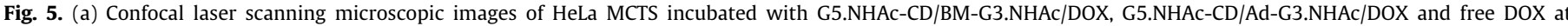

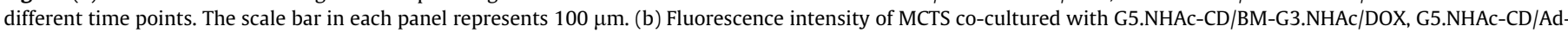

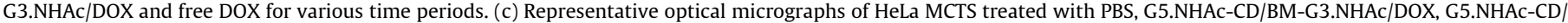

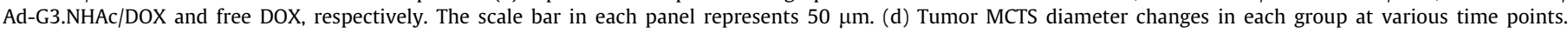
Reproduced with permission from ref. [96], copyright 2019, Royal Society of Chemistry.

tration and cellular uptake ability as compared to free DOX and pH-insensitive CSTDs/DOX (G5.NHAc-CD/Ad-G3.NHAc/DOX) complexes (Fig. 5a,b). On the other hand, through measurements of the size of 3D MCTS (Fig. 5c,d), we found that the inhibition efficiency followed the order of $\mathrm{pH}$-responsive CSTDs/DOX group $(26.7 \%)>$ free DOX $(19.2 \%)>$ pH-insensitive CSTDs/DOX group (13.5\%) within 7 days of treatment.

Efficient drug delivery vehicles should possess favorable drug loading and releasing capabilities [13,116]. Unlike the CSTDs with limited drug loading capacity, dendrimer NGs with a larger dimension can be regarded as nanocarriers with an enhanced drug loading capacity and efficiency [65]. For instance, AG-G5 NGs could be loaded with anticancer drug EPI with a loading efficiency of $93.3 \pm 2.22 \%$ for chemotherapy of cancer cells [69]. The obtained EPI $\in$ AG-G5 NGs could release EPI in a sustained manner with a faster EPI release at pH 5.5 than $\mathrm{pH}$ 7.4. Within 48 h, 47.6\% EPI was released at $\mathrm{pH} 5.5$, while $37.2 \%$ of EPI was released in PBS ( $\mathrm{pH}$ 7.4). What is more, the EPI $\in$ AG-G5 NGs could not only be uptaken and sufficiently accumulated in MCF-7 cells in a doseand time-dependent manner, but also induced effective cancer cell death.

To render the carrier system with the cellular environmentresponsive drug delivery, enzyme-responsive dendrimer NGs were prepared for drug delivery applications [57]. The dendrimer NGs displayed good biocompatibility, five times higher DOX loading ability (6.93 $\pm 1.55 \%)$ than dendrimer macromonomer (1.48 \pm 0.5 $9 \%$ ), as well as enzyme-responsive drug release property due to the incorporation of the enzyme-sensitive peptide sequence. The enzyme-triggered rapid drug release within the cells rendered the therapeutic activity of DOX-loaded dendrimer NGs to be as high as that of free DOX, which is very important for effective cancer chemotherapy.

For chemotherapy, besides CSTDs and dendrimer NGs, dendrimer-templated hybrid NCs have also been employed as an effective nanoplatform. As depicted in Fig. 6, Li et al. [60] developed a strategy to establish the $\mathrm{pH}$-sensitive clustered nanobombs for improved drug penetration in tumors and chemotherapeutic efficacy. In this case, dendrimers were first conjugated with Pt prodrug. Separately, pH-sensitive amphiphilic block copolymer (PEG-b-poly(2-azepane ethyl methacrylate), for short, PEG-bPAEMA) was obtained by reversible addition-fragmentation chain transfer polymerization, reduced by 2 -aminoethanol to produce sulfhydryl-terminated PEG-b-PAEMA and modified with $\mathrm{N}-(\varepsilon$-mal eimidocaproyloxy)-succinimide ester (NHS). The resulting PEG-bPAEMA-NHS was coupled with PAMAM/Pt to compose PEG-bPAEMA-PAMAM/Pt. Finally, the pH-sensitive clustered nanobombs were created via self-assembly of PEG-bPAEMA-PAMAM/Pt. The $\mathrm{pH}$-responsive NCs could have prolonged blood circulation time in vivo because of their large size, thereby increasing the propensity of large NPs to extravasate from leaky tumor vasculature to accumulate in the vicinity of blood vessels. In the vascular microenvironment $(\mathrm{pH} 7.4)$, the $\mathrm{pH}$-sensitive clustered nanobombs were stable, while at acidic tumor $\mathrm{pH}(\mathrm{pH} \sim 6.5-7.0)$, they would instantaneously switch to small particles $(<10 \mathrm{~nm})$ for deep tumor penetration and to release the Pt drug for effective cancer chemotherapy. Similarly, Li et al. [59] also prepared Pt-containing iCluster that had improved tumor accumulation through the passive EPR effect and enabled prodrugs to reach evenly inside the tumors via $\mathrm{pH}$-responsive fast release to kill cancer cells effectively.

\subsection{Combination therapy and theranostics}

The treatment of malignant tumors using single-mode therapeutic approach always displays limited outcomes because of its inherent imperfection $[117,118]$. Therefore, it is necessary to propose combination therapy approaches to enhance tumor therapeutic efficacy. SDNs have also been used as a carrier system for combination therapy. For example, through conjugating branched PEG with G2 dendrimers, Wang et al. [97] prepared a type of structure-invertible SDNs for co-delivery of nucleic acids and hydrophobic drugs. In their study, the cleavage of disulfide link- 
(a)
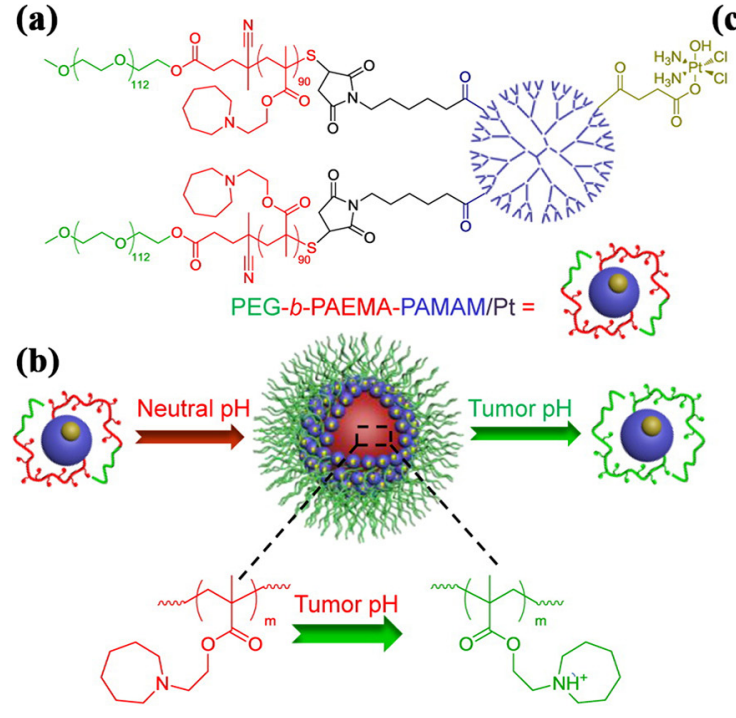

(c)

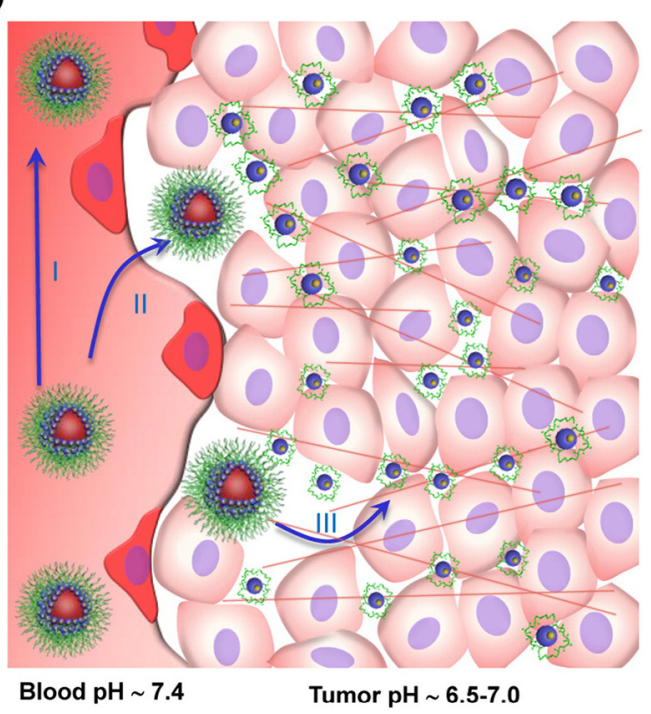

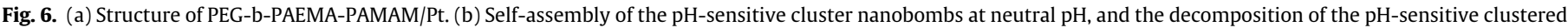

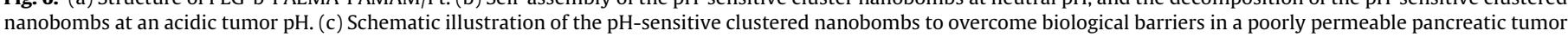
model. Reproduced with permission from ref. [60], copyright 2010, American Chemical Society.

ages at intracellular glutathione-rich environment significantly decreased the cytotoxicity of the SDNs, and promoted highly efficient drug release and gene transfection in vitro and in vivo. Compared to free $\mathrm{G} 2$ dendrimers, this nanocarrier not only displayed an enhanced endosomal escape capability and improved serum stability in vitro, but also exhibited prolonged residence time and stronger transfection activity in vivo. Most importantly, co-delivery of DOX and B-cell lymphoma 2 (Bcl-2) small interfering RNA (siRNA) exerted a combinational effect against tumor growth in a murine tumor model in vivo, which was much more effective than either DOX or Bcl-2 siRNA-based monotherapy. In our recent study, in order to fully use the large interior space and the excellent gene compact ability of the CSTDs, we reported the use of G5-CD/AdG3 CSTDs to co-deliver therapeutic miRNA 21 inhibitor and anticancer drug DOX for enhanced cancer therapy applications [67]. We showed that the transfection efficiency of CSTDs/miR 21i polyplexes was the highest at the N/P ratio of 10, leading to effective regulation of the target genes/proteins and inhibition of cancer cell migration. More importantly, co-delivery of miR21i and DOX using CSTDs as a vector could result in more significant therapeutic efficacy of cancer cells than that of DOX only. In another report [100], dendrimer NGs constructed via host-guest interaction between Ad-conjugated random copolymers and $\beta$-CD-modified G3 PAMAM dendrimers were also used to simultaneously encapsulate both DOX for chemotherapy and indocyanine green (ICG) for photothermal therapy by electrostatic interaction. The release of DOX from the NGs could be triggered by NIR light to lead to NG dissociation. The cytotoxicity evaluation results revealed that the DOX $\mathrm{IC}_{50}$ of the ICG-G3-DOX NGs decreased significantly from $33 \mu \mathrm{g} / \mathrm{mL}$ to $7 \mu \mathrm{g} / \mathrm{mL}$ under NIR irradiation, suggesting the cooperative effect exerted to cancer cells. Furthermore, after a total of three intravenous injections (day 0,2 , and 4 ) and three NIR irradiations (day 1,3 , and 5), the in vivo antitumor efficacy assay data displayed that the control groups of PBS and free DOX did not have statistically significant differences in tumor growth suppression. The group of ICG-G3-DOX NGs with NIR laser irradiation was much more effective in killing tumors than the groups of ICG-G3-DOX NGs without NIR laser irradiation and G3-DOX NGs with NIR laser irradiation.

In the field of precision or personalized medicine, imagingguided cancer therapy has been emerging as a new and efficient strategy to tackle cancer [119], which is currently a hotspot in cancer nanomedicine. SDNs-based nanoplatforms also hold great promise for cancer theranostics by integrating both imaging elements and therapeutic components. As a representative example, Goncalves et al. [68] reported the G5 dendrimer-assisted formation of AG NGs for fluorescence imaging and chemotherapy of cancer cells in vitro. In their study, FITC-labeled G5 dendrimers were integrated into the AG NGs as an additional crosslinker besides $\mathrm{Ca}^{2+}$-induced crosslinking for subsequent encapsulation of DOX. Such incorporation of the dendrimers rendered the NGs with a loading capacity 3 times higher than dendrimer-free AG NGs. The AG-G5 NGs could release DOX in a sustained manner, be taken up by cancer cells to exert the DOX-associated anticancer cytotoxicity, and be fluorescence imaged owing to the conjugated fluorescent marker of FITC. These findings demonstrated that the developed AG-G5 NGs might serve as a general nanoplatform for fluorescence imagingguided therapeutic delivery to cancer cells in vitro and in vivo.

\section{Conclusion and outlooks}

In summary, this review has described the recent developments of different SDNs as nanoplatforms for cancer nanomedicine applications. Through the unique designs, DDs, CSTDs, dendrimer NCs, dendrimer NGs, and hybrid dendrimer-templated NCs can be prepared to have a variety of different supramolecular structures. Compared to individual dendrimer-based platforms, more functionalities or desired properties of SDNs can be achieved to have enhanced EPR-based tumor passive targeting, stimuliresponsiveness, and improved drug loading and imaging sensitivity (e.g., improved $r_{1}$ relaxivity for MR imaging purpose). With these rendered important advantages, the SDNs with controlled structure and stability can be designed for improved cancer nanomedicine applications including bioimaging, gene delivery, chemotherapy, combination therapy, and theranostics to tackle some key problems related to cancer imaging and therapy.

Despite numerous attempts and inspiring results achieved so far, much room remains to be explored and addressed to further improve the cancer nanomedicine applications of SDNs. For instance, currently, the SDNs are only designed to be $\mathrm{pH}-$, enzyme- or redox-responsive, other stimuli-responsive SDNs or 
dual-/multi-responsive SDNs may be further designed to meet the tumor microenvironment for better imaging and therapy. For combination therapy, more therapeutic modes or combinations incorporated with immunotherapy, radiotherapy or others should be further explored to improve the tumor treatment outcomes. In addition, for precision imaging of tumors, the SDNs could be further designed to incorporate different imaging elements for dualmode or multi-mode imaging purposes. Furthermore, approaches to scale up production of the desired SDNs with quality control and further studies related to pharmacokinetic, pharmacodynamics, metabolism, biodistribution, and long-term toxicity should also be performed to shine up the promising potential of SDNs for cancer nanomedicine applications.

\section{Declaration of Competing Interest}

The authors declare that they have no known competing financial interests or personal relationships that could have appeared to influence the work reported in this paper.

\section{Acknowledgements}

This research has been financially supported by the National Key R\&D Program (2017YFE0196200), the National Natural Science Foundation of China (21911530230, 81761148028 and 21773026), the Science and Technology Commission of Shanghai Municipality (19XD1400100 and 19410740200), and the Fundamental Research Funds for the Central Universities (CUSF-DH-D-2019073). S.M. and J.P.M. thank the NSFC-CNRS collaboration project (199675). J.R., S. M. and X.S. acknowledge the support of FCT-Fundação para a Ciência e a Tecnologia (Base Fund UIDB/00674/2020 and Programmatic Fund UIDP/00674/2020, Portuguese Government Funds) and ARDITI-Agência Regional para o Desenvolvimento da Investigação Tecnologia e Inovação through the project M1420-01-0145FEDER-000005-CQM ${ }^{+}$(Madeira 14-20 Program), Portugal. J. P. Majoral thanks CNRS (France) for financial support.

\section{References}

[1] D.A. Tomalia, H. Baker, J. Dewald, M. Hall, G. Kallos, S. Martin, J. Roeck, J. Ryder, P. Smith, Polym. J. 17 (1985) 117-132.

[2] D.A. Tomalia, A.M. Naylor, W.A. Goddard, Angew. Chem., Int. Ed. 29 (1990) $138-175$.

[3] B. Helms, E.W. Meijer, Science 313 (2006) 929-930.

[4] D.A. Tomalia, Prog. Polym. Sci. 30 (2005) 294-324.

[5] C.J. Hawker, J.M.J. Frechet, J. Am. Chem. Soc. 112 (1990) 7638-7647.

[6] K. Karatasos, D.B. Adolf, G.R. Davies, J. Chem. Phys. 115 (2001) 5310-5318.

[7] Y. Cheng, L. Zhao, Y. Li, T. Xu, Chem. Soc. Rev. 40 (2011) 2673-2703.

[8] S. Sadekar, H. Ghandehari, Adv. Drug Deliv. Rev. 64 (2012) 571-588.

[9] S. Mignani, J. Rodrigues, H. Tomas, M. Zablocka, X. Shi, A.-M. Caminade, J.-P. Majoral, Chem. Soc. Rev. 47 (2018) 514-532.

[10] A.P. Dias, S.d.S. Santos, J.V. da Silva, R. Parise-Filho, E.I. Ferreira, O. El Seoud, J. Giarolla, Int. J. Pharm. 573 (2020) 118814.

[11] Z. Xiong, M. Shen, X. Shi, Sci. China Mater. 61 (2018) 1387-1403.

[12] Y. Fan, W. Sun, X. Shi, Small Methods 1 (2017) 1700234

[13] J. Zhu, X. Shi, J. Mater. Chem. B 1 (2013) 4199-4211.

[14] X. He, C.S. Alves, N. Oliveira, J. Rodrigues, J. Zhu, I. Banyai, H. Tomas, X. Shi, Colloids Surf., B 125 (2015) 82-89.

[15] W. Hou, P. Wei, L. Kong, R. Guo, S. Wang, X. Shi, J. Mater. Chem. B 4 (2016) 2933-2943.

[16] L. Kong, C.S. Alves, W. Hou, J. Qiu, H. Moehwald, H. Tomas, X. Shi, ACS Appl Mater. Interfaces 7 (2015) 4833-4843.

[17] L. Lin, Y. Fan, F. Gao, L. Jin, D. Li, W. Sun, F. Li, P. Qin, Q. Shi, X. Shi, L. Du, Theranostics 8 (2018) 1923-1939.

[18] J. Qiu, L. Kong, X. Cao, A. Li, H. Tan, X. Shi, RSC Adv. 6 (2016) 25633-25640.

[19] Y. Shan, T. Luo, C. Peng, R. Sheng, A. Cao, X. Cao, M. Shen, R. Guo, H. Tomas, X. Shi, Biomaterials 33 (2012) 3025-3035.

[20] W. Sun, S. Mignani, M. Shen, X. Shi, Drug Discovery Today 21 (2016) 1873 1885.

[21] T. Xiao, D. Li, X. Shi, M. Shen, Macromol. Biosci. 20 (2020) 1900282.

[22] S. Wen, L. Zhao, Q. Zhao, D. Li, C. Liu, Z. Yu, M. Shen, J.-P. Majoral, S. Mignani, J. Zhao, X. Shi, J. Mater. Chem. B 5 (2017) 3810-3815.

[23] J. Zhu, L. Zhao, Y. Cheng, Z. Xiong, Y. Tang, M. Shen, J. Zhao, X. Shi, Nanoscale 7 (2015) 18169-18178.
[24] L. Zhao, J. Zhu, Y. Cheng, Z. Xiong, Y. Tang, L. Guo, X. Shi, J. Zhao, ACS Appl. Mater. Interfaces 7 (2015) 19798-19808.

[25] Z. Qiao, X. Shi, Prog. Polym. Sci. 44 (2015) 1-27.

[26] J. Liu, Z. Xiong, J. Zhang, C. Peng, B. Klajnert-Maculewicz, M. Shen, X. Shi, ACS Appl Mater. Interfaces 11 (2019) 15212-15221.

[27] S. Lu, X. Li, J. Zhang, C. Peng, M. Shen, X. Shi, Adv. Sci. 5 (2018) 1801612.

[28] X. Xu, K. Liu, Y. Wang, C. Zhang, M. Shi, P. Wang, L. Shen, J. Xia, L. Ye, X. Shi, M. Shen, J. Mater. Chem. B 7 (2019) 3639-3643.

[29] J. Zhu, G. Wang, C.S. Alves, H. Tomas, Z. Long, M. Shen, J. Rodrigues, X. Shi, Langmuir 34 (2018) 12428-12435.

[30] Y. Xiao, X. Shi, Nanomedicine 14 (2019) 2515-2518.

[31] X. Li, Z. Xiong, X. Xu, Y. Luo, C. Peng, M. Shen, X. Shi, ACS Appl. Mater Interfaces 8 (2016) 19883-19891.

[32] Z. Xiong, Y. Wang, J. Zhu, X. Li, Y. He, J. Qu, M. Shen, J. Xia, X. Shi, Nanoscale 9 (2017) 12295-12301.

[33] L. Zhao, X. Shi, J. Zhao, Drug Delivery 24 (2017) 81-93.

[34] H. Cai, K. Li, J. Li, S. Wen, Q. Chen, M. Shen, L. Zheng, G. Zhang, X. Shi, Small 11 (2015) 4584-4593.

[35] J. Zhu, L. Zheng, S. Wen, Y. Tang, M. Shen, G. Zhang, X. Shi, Biomaterials 35 (2014) 7635-7646.

[36] Y. Fan, J. Zhang, M. Shi, D. Li, C. Lu, X. Cao, C. Peng, S. Mignani, J.-P. Majoral, X Shi, Nano Lett. 19 (2019) 1216-1226.

[37] Y. Fan, W. Tu, M. Shen, X. Chen, Y. Ning, J. Li, T. Chen, H. Wang, F. Yin, Y. Liu, X Shi, Adv. Funct. Mater. 30 (2020) 1909285.

[38] P. Wei, J. Chen, Y. Hu, X. Li, H. Wang, M. Shen, X. Shi, Adv. Healthcare Mater. 5 (2016) 3203-3213.

[39] J.F. Kukowska-Latallo, A.U. Bielinska, J. Johnson, R. Spindler, D.A. Tomalia, J.R. Baker Jr., Proc. Natl. Acad. Sci. U. S. A. 93 (1996) 4897-4902.

[40] Y. Cheng, Y. Li, Q. Wu, J. Zhang, T. Xu, Eur. J. Med. Chem. 44 (2009) 2219-2223.

[41] S. Hong, P.R. Leroueil, E.K. Janus, J.L. Peters, M.-M. Kober, M.T. Islam, B.G. Orr, J.R. Baker Jr., M.M.B. Holl, Bioconjugate Chem. 17 (2006) 728-734.

[42] S. Hong, R. Rattan, I.J. Majoros, D.G. Mullen, J.L. Peters, X. Shi, A.U. Bielinska, L. Blanco, B.G. Orr, J.R. Baker Jr., M.M.B. Holl, Bioconjugate Chem. 20 (2009) 1503-1513.

[43] N. Malik, R. Wiwattanapatapee, R. Klopsch, K. Lorenz, H. Frey, J.W. Weener, E W. Meijer, W. Paulus, R. Duncan, J. Controlled Release 68 (2000) 299-302.

[44] Z. Xiong, Y. Wang, J. Zhu, Y. He, J. Qu, C. Effenberg, J. Xia, D. Appelhans, X. Shi, Biomater. Sci. 4 (2016) 1622-1629.

[45] J. Zhu, Z. Xiong, M. Shen, X. Shi, RSC Adv. 5 (2015) 30286-30296.

[46] X.Y. Shi, I. Banyai, M.T. Islam, W. Lesniak, D.Z. Davis, J.R. Baker Jr., L.P. Balogh, Polymer 46 (2005) 3022-3034.

[47] M.K. LothianTomalia, D.M. Hedstrand, D.A. Tomalia, A.B. Padias, H.K. Hall Tetrahedron 53 (1997) 15495-15513.

[48] A.R. Menjoge, R.M. Kannan, D.A. Tomalia, Drug Discovery Today 15 (2010) $171-185$.

[49] Y.S. Choi, A. Mecke, B.G. Orr, M.M.B. Holl, J.R. Baker Jr., Nano Lett. 4 (2004) 391-397.

[50] Y. Choi, T. Thomas, A. Kotlyar, M.T. Islam, J.R. Baker Jr., Chem. Biol. 12 (2005) 35-43.

[51] J. Li, D.R. Swanson, D. Qin, H.M. Brothers, L.T. Piehler, D. Tomalia, D.J. Meier, Langmuir 15 (1999) 7347-7350.

[52] S. Uppuluri, D.R. Swanson, L.T. Piehler, J. Li, G.L. Hagnauer, D.A. Tomalia, Adv. Mater. 12 (2000) 796-800.

[53] D.A. Tomalia, H.M. Brothers, L.T. Piehler, H.D. Durst, D.R. Swanson, Proc. Natl. Acad. Sci. U. S. A. 99 (2002) 5081-5087.

[54] Z. Cheng, D.L.J. Thorek, A. Tsourkas, Angew. Chem., Int. Ed. 49 (2010) 346350.

[55] C.-H. Huang, K. Nwe, A. Al Zaki, M.W. Brechbiel, A. Tsourkas, ACS Nano 6 (2012) 9416-9424.

[56] H. Liu, H. Wang, W. Yang, Y. Cheng, J. Am. Chem. Soc. 134 (2012) 17680 17687.

[57] Y. Wang, Y. Luo, Q. Zhao, Z. Wang, Z. Xu, X. Jia, ACS Appl. Mater. Interfaces 8 (2016) 19899-19906.

[58] D. Zhang, P.D. Hamilton, J.L.F. Kao, S. Venkataraman, K.L. Wooley, N. Ravi, J. Polym. Sci., Part A: Polym. Chem. 45 (2007) 2569-2575.

[59] H.-J. Li, J.-Z. Du, X.-J. Du, C.-F. Xu, C.-Y. Sun, H.-X. Wang, Z.-T. Cao, X.-Z. Yang, Y.-H. Zhu, S. Nie, J. Wang, Proc. Natl. Acad. Sci. U. S. A. 113 (2016) 4164-4169.

[60] H.-J. Li, J.-Z. Du, J. Liu, X.-J. Du, S. Shen, Y.-H. Zhu, X. Wang, X. Ye, S. Nie, J. Wang, ACS Nano 10 (2016) 6753-6761.

[61] H. Wang, S. Wang, H. Su, K.-J. Chen, A.L. Armijo, W.-Y. Lin, Y. Wang, J. Sun, K.-I. Kamei, J. Czernin, C.G. Radu, H.-R. Tseng, Angew. Chem., Int. Ed. 48 (2009) 4344-4348.

[62] J. Wang, I.K. Voets, R. Fokkink, J. van der Gucht, A.H. Velders, Soft Matter 10 (2014) 7337-7345.

[63] F. Chen, L. Kong, L. Wang, Y. Fan, M. Shen, X. Shi, J. Mater. Chem. B 5 (2017) 8459-8466.

[64] S. Lu, X. Bao, W. Hai, S. Shi, Y. Chen, Q. Yu, M. Zhang, Y. Xu, J. Peng, Int. J Pharm. 575 (2020) 118898

[65] L. Phung Ngan, N. Ngoc Hoa, N. Cuu Khoa, T. Ngoc Quyen, Bull. Mater. Sci. 39 (2016) 1493-1500.

[66] P. Schilrreff, G. Cervini, E.L. Romero, M.J. Morilla, Colloids Surf., B 122 (2014) 19-29.

[67] Cong Song, Yunchao Xiao, Zhijun Ouyang, Mingwu Shen, X. Shi, J. Mater Chem. B 8 (2020) 2768-2774.

[68] M. Goncalves, D. Maciel, D. Capelo, S. Xiao, W. Sun, X. Shi, J. Rodrigues, H. Tomas, Y. Li, Biomacromolecules 15 (2014) 492-499. 
[69] I. Matai, P. Gopinath, ACS Biometer. Sci. Eng. 2 (2016) 213-223.

[70] X. Xu, Y. Jian, Y. Li, X. Zhang, Z. Tu, Z. Gu, ACS Nano 8 (2014) 9255-9264.

[71] D.Q. McNerny, J.F. Kukowska-Latallo, D.G. Mullen, J.M. Wallace, A.M. Desai, R. Shukla, B.H. Huang, M.M.B. Holl, J.R. Baker Jr., Bioconjugate Chem. 20 (2009) 1853-1859.

[72] X.X. Liu, C. Liu, J.H. Zhou, C. Chen, F.Q. Qu, J.J. Rossi, P. Rocchi, L. Peng, Nanoscale 7 (2015) 3867-3875.

[73] T. Yu, X. Liu, A.-L. Bolcato-Bellemin, Y. Wang, C. Liu, P. Erbacher, F. Qu, P. Rocchi, J.-P. Behr, L. Peng, Angew. Chem., Int. Ed. 51 (2012) 8478-8484.

[74] J.J. Xiang, B.H. Wu, Z.X. Zhou, S.Q. Hu, Y. Piao, Q. Zhou, G.W. Wang, J.B. Tang, X. R. Liu, Y.Q. Shen, Sci. China Life Sci. 61 (2018) 436-447.

[75] M. Shen, X. Shi, Nanoscale 2 (2010) 1596-1610.

[76] X. Shi, K. Sun, J.R. Baker Jr., J. Phys. Chem. C 112 (2008) 8251-8258.

[77] X. Shi, S.H. Wang, M.E. Van Antwerp, X. Chen, J.R. Baker Jr., Analyst 134 (2009) 1373-1379.

[78] H. Liu, M. Shen, J. Zhao, R. Guo, X. Cao, G. Zhang, X. Shi, Colloid Surf. BBiointerfaces 94 (2012) 58-67.

[79] H. Liu, M. Shen, J. Zhao, J. Zhu, T. Xiao, X. Cao, G. Zhang, X. Shi, Analyst 138 (2013) 1979-1987.

[80] H. Liu, K. Sun, J. Zhao, R. Guo, M. Shen, X. Cao, G. Zhang, X. Shi, Colloid Surf. APhysicochem. Eng. Asp. 405 (2012) 22-29.

[81] H. Liu, H. Wang, R. Guo, X. Cao, J. Zhao, Y. Luo, M. Shen, G. Zhang, X. Shi, Polym. Chem. 1 (2010) 1677-1683.

[82] H. Liu, Y. Xu, S. Wen, Q. Chen, L. Zheng, M. Shen, J. Zhao, G. Zhang, X. Shi, Chem. Eur. J. 19 (2013) 6409-6416.

[83] H. Liu, Y. Xu, S. Wen, J. Zhu, L. Zheng, M. Shen, J. Zhao, G. Zhang, X. Shi, Polym. Chem. 4 (2013) 1788-1795.

[84] C. Peng, K. Li, X. Cao, T. Xiao, W. Hon, L. Zheng, R. Guo, M. Shen, G. Zhang, X. Shi, Nanoscale 4 (2012) 6768-6778.

[85] T. Xiao, J. Qin, C. Peng, R. Guo, X. Lu, X. Shi, Langmuir 36 (2020) 3096-3103.

[86] X. Shi, S.H. Wang, M. Shen, M.E. Antwerp, X. Chen, C. Li, E.J. Petersen, Q. Huang, W.J. Weber Jr., J.R. Baker Jr., Biomacromolecules 10 (2009) 17441750.

[87] X. Shi, S.H. Wang, S.D. Swanson, S. Ge, Z. Cao, M.E. Van Antwerp, K.J. Landmark, J.R. Baker Jr., Adv. Mater. 20 (2008) 1671-1678.

[88] S.H. Wang, X. Shi, M. Van Antwerp, Z. Cao, S.D. Swanson, X. Bi, J.R. Baker Jr., Adv. Funct. Mater. 17 (2007) 3043-3050.

[89] H. Cai, K. Li, M. Shen, S. Wen, Y. Luo, C. Peng, G. Zhang, X. Shi, J. Mater. Chem. 22 (2012) 15110-15120.

[90] L. Kong, L. Xing, B. Zhou, L. Du, X. Shi, ACS Appl. Mater. Interfaces 9 (2017) 15995-16005.

[91] N. Katir, N. El Brahmi, N. Marcotte, J.P. Majoral, M. Bousmina, A. El Kadib, Macromolecules 49 (2016) 5796-5805.

[92] C.R. DeMattei, B.H. Huang, D.A. Tomalia, Nano Lett. 4 (2004) 771-777.

[93] Y. Choi, J.R. Baker Jr., Cell Cycle 4 (2005) 669-671.
[94] T. Mandal, M.V.S. Kumar, P.K. Maiti, J. Phys. Chem. B 118 (2014) 1180511815.

[95] P. Schilrreff, C. Mundina-Weilenmann, E. Lilia Romero, M. Jose Morilla, Int. J. Nanomed. 7 (2012) 4121-4133.

[96] J. Wang, D. Li, Y. Fan, M. Shi, Y. Yang, L. Wang, Y. Peng, M. Shen, X. Shi, Nanoscale 11 (2019) 22343-22350.

[97] K. Wang, Q. Hu, W. Zhu, M. Zhao, Y. Ping, G. Tang, Adv. Funct. Mater. 25 (2015) 3380-3392.

[98] M.R. Kotte, A.T. Kuvarega, S.N. Talapaneni, M. Cho, A. Coskun, M.S. Diallo, ACS Appl. Mater. Interfaces 10 (2018) 33238-33251.

[99] H. Wang, Y. Wang, Y. Wang, J. Hu, T. Li, H. Liu, Q. Zhang, Y. Cheng, Angew. Chem., Int. Ed. 54 (2015) 11647-11651.

[100] M.H. Zan, J.J. Li, M.M. Huang, S.Q. Lin, D. Luo, S.Z. Luo, Z.S. Ge, Biomater. Sci. 3 (2015) 1147-1156.

[101] J.B. ten Hove, J. Wang, F.W.B. van Leeuwen, A.H. Velders, Nanoscale 9 (2017) 18619-18623.

[102] J.B. ten Hove, M.N. van Oosterom, F.W.B. van Leeuwen, A.H. Velders, Sci. Rep. 8 (2018) 13820

[103] K. McCoy, M. Uchida, B. Lee, T. Douglas, ACS Nano 12 (2018) 3541-3550.

[104] H. Wang, K.-J. Chen, S. Wang, M. Ohashi, K.-I. Kamei, J. Sun, J.H. Ha, K. Liu, H.R. Tseng, Chem. Commun. 46 (2010) 1851-1853.

[105] S. Mirtschin, A. Slabon-Turski, R. Scopelliti, A.H. Velders, K. Severin, J. Am. Chem. Soc. 132 (2010) 14004-14005.

[106] B.L. Frankamp, A.K. Boal, V.M. Rotello, J. Am. Chem. Soc. 124 (2002) 15146 15147.

[107] P.-C. Chen, G. Liu, Y. Zhou, K.A. Brown, N. Chernyak, J.L. Hedrick, S. He, Z. Xie, Q.-Y. Lin, V.P. Dravid, S.A. O'Neill-Slawecki, C.A. Mirkin, J. Am. Chem. Soc. 137 (2015) 9167-9173.

[108] M.Y. Berezin, S. Achilefu, Chem. Rev. 110 (2010) 2641-2684.

[109] T.F. Massoud, S.S. Gambhir, Genes Dev. 17 (2003) 545-580.

[110] S.P. Chaplot, I.D. Rupenthal, J. Pharm. Pharmacol. 66 (2014) 542-556.

[111] L. Jiang, S. Zhou, X. Zhang, W. Wu, X. Jiang, Sci. China Mater. 61 (2018) 14041419.

[112] K.L. Killops, L.M. Campos, C.J. Hawker, J. Am. Chem. Soc. 130 (2008) 50625064.

[113] R.-Q. Huang, Y.-H. Qu, W.-L. Ke, J.-H. Zhu, Y.-Y. Pei, C. Jiang, FASEB J. 21 (2007) $1117-1125$.

[114] S. Wang, F. Wang, Q. Zhang, Y. Cheng, J. Mater. Chem. B 5 (2017) 5101-5108.

[115] L. Kong, Y. Wu, C.S. Alves, X. Shi, Nanomedicine 11 (2016) 3103-3115.

[116] X. Li, M. Takashima, E. Yuba, A. Harada, K. Kono, Biomaterials 35 (2014) 6576-6584.

[117] E. Blanco, A. Hsiao, A.P. Mann, M.G. Landry, F. Meric-Bernstam, M. Ferrari, Cancer Sci. 102 (2011) 1247-1252.

[118] S. Kala, A.S.C. Mak, X. Liu, P. Posocco, S. Pricl, L. Peng, A.S.T. Wong, J. Med. Chem. 57 (2014) 2634-2642.

[119] L. Chin, J.N. Andersen, P.A. Futreal, Nat. Med. 17 (2011) 297-303. 